\title{
Effects of sustained, voluntary attention on amplitude and latency of steady-state visual evoked potential: a costs and benefits analysis
}

\author{
F. Di Russo ${ }^{\mathrm{a}, *}$, D. Spinelli ${ }^{\mathrm{a}, \mathrm{b}}$ \\ ${ }^{\text {a }}$ Fondazione Santa Lucia, IRCCS (Centro Ricerche di Neuropsicologia), via Ardeatina 306, 00179 Rome, Italy \\ ${ }^{\mathrm{b}}$ Istituto Universitario Scienze Motorie, Rome, Italy
}

Accepted 22 July 2002

\begin{abstract}
Objective: Steady-state visual evoked potentials (VEPs) were recorded to study the mechanisms that underlie visual attention.

Methods: VEPs were recorded from 1 cycle/degree sinusoidal grating contrast reversed at various temporal frequencies (6-10 Hz). This was displayed in one hemifield. A letter search display was flashed at a random rate in the other hemifield. The subject performed a demanding task on the recording stimulus (attended condition) or on the opposite side stimulus (unattended condition). Alternatively, he/ she passively fixated on the fixation point (passive condition).

Results: Relative to the passive condition, attended stimuli elicited enhanced-amplitude and shortened-latency VEP (benefits). Costs (i.e. responses to passive vs. unattended stimuli) were more marked for latency.

Conclusions: VEP latency may be the key of a priority-based attention mechanism acting at an early level. (C) 2002 Elsevier Science Ireland Ltd. All rights reserved.
\end{abstract}

Keywords: Steady-state visual evoked potential; Visual attention; Temporal frequency

\section{Introduction}

The neural mechanisms of attention have been studied extensively using transient visual evoked potentials (VEPs) (for a review, see Hillyard et al., 1995; Martínez et al., 2001b). Attention to a specific region of the visual field is associated with increased amplitudes of VEP components. The components most consistently enhanced are the P1 (peak latency 80-130 ms) and the N1 (peak latency 140$200 \mathrm{~ms}$ ) waves (e.g. Hillyard et al., 1998). It has been proposed that such attentional modulation reflects a sensory gain control mechanism, whereby visual information falling within the spotlight of spatial attention is facilitated and passed along to higher levels of processing (Hillyard and Mangun, 1987; Hillyard and Annlo-Vento, 1998). Attentional modulation of this latter processing is indexed by long-latency visual event related potentials such as the posteriorly distributed N1 component (140-200 ms), the selection negativity (150-300 ms) and the N2 (200-300 $\mathrm{ms})$ and P3 (300-500 ms) components (e.g. Vogel and Luck, 2000; Müller and Hillyard, 2000).

\footnotetext{
* Corresponding author. Tel.: +39-06-5150-1358; fax: +39-06-51501366.

E-mail address: fdirusso@uniroma1.it (F. Di Russo).
}

On the other hand, the effects of attention on steady-state VEPs have been less extensively studied (Morgan et al., 1996; Müller et al., 1998a,b; Di Russo and Spinelli, 1999a,b; Müller and Hillyard, 2000). In these experiments, VEP was compared in two conditions (attended vs. unattended). In the attended condition, VEP was recorded from a lateral task-irrelevant background while the subject attended a demanding task on a visual object superimposed on the background. In the unattended condition, the subject performed the same task on the opposite side. Confirming data obtained with the transient technique, spatial attention related enhancement of steady-state VEP amplitude was shown in all studies. Further, two studies (Di Russo and Spinelli, 1999a,b) showed that attention also decreased the 'apparent latency' (Spekreijse et al., 1977; Regan, 1989) of the evoked responses. VEP in the attended condition had a shorter 'apparent latency' (hereafter called 'latency') than VEP in the unattended condition. The latency difference produced by attention ranged from 5 to $20 \mathrm{~ms}$.

VEP latency modulation by attention may have an important functional meaning. If attention is a mechanism that provides priority for perception, consciousness and action (Posner, 1995), shortening of latency at an early stage can be a good tool to provide priority for the brain signal evoked by the attended stimulus. Moreover, we noted (Di Russo and 
Spinelli, 1999a) that changes in amplitude and latency are compatible with the description of automatic gain control mechanisms acting on early visual processing (e.g. Shapley and Victor, 1978; Bernardete et al., 1992). The latter reasoning was developed by measuring the effect of attention on the evoked response at different levels of stimulus contrast (Di Russo et al., 2001a). Consistent with models for the mediation of contrast gain control (Shapley and Victor, 1981; Carandini and Heeger, 1994), attention increased VEP amplitudes and reduced the phase-advance with contrast. Thus, it seems that attention may increase the efficiency in processing time and contrast discriminability of a stimulus by using the same mechanism already in place for the basic sensory function of contrast regulation (Di Russo et al., 2001a).

In the latter research, as well as in the present study, we modified the experimental paradigm with respect to the previously cited studies. In the attended condition, the subject paid attention to a grating displayed on one side and discriminated the contrast changes (Di Russo et al., 2001a) or the temporal frequency changes (present study). Thus, the evoking stimulus was not only an irrelevant background located on the attended space as in the earlier studies, but also the target on which the subject performed a demanding discrimination task.

Here, we compared the attended responses with those recorded when the subject was involved in a highly demanding task on the stimulus displayed on the opposite side, and with a neutral condition in which the subject's only task was to fixate on the central fixation point. This latter condition is instrumental for calculating attentional benefits (i.e. differences between attended and neutral conditions) and costs (i.e. differences between neutral and unattended conditions) on VEP amplitude and latency (Proverbio and Mangun, 1994; Eimer, 1996; Mangun and Buck, 1999).

\section{Methods}

\subsection{Subjects}

Thirteen neurologically normal subjects ( 8 females; mean age 26.7 years) were studied. All subjects had normal or correct-to-normal vision. Subjects were informed of the general purpose of the experiment to insure maximum reliability in the fixation task.

\subsection{Stimuli}

Steady-state VEPs were recorded from horizontally oriented contrast-reversal sinusoidal gratings. The grating (1 cycle/degree spatial frequency, subtending $9 \times 9^{\circ}$ of visual field) was continuously displayed in the left visual field (LVF). The inner edge of the grating had an eccentricity of $3^{\circ}$ from the fixation point. The grating contrast was modulated in luminance (yellow-black) with $20 \%$ contrast. The mean luminance was $16 \mathrm{~cd} / \mathrm{m}^{2}$. The contrast of the grating was reversed with sinusoidal temporal frequency varying between 6 and $10 \mathrm{~Hz}$ (step $0.5 \mathrm{~Hz}$ ). Temporal frequency changes occurred randomly about 20 times during each recording sequence.

A $3 \times 3$ letters array was flashed in the right visual field (RVF). The array subtended $3 \times 3^{\circ}$, with each letter subtending $0.5^{\circ}$. The inner border had an eccentricity of $3^{\circ}$ from the fixation point. The distracter letters were F; one target letter $\mathrm{O}$ was presented among them in half of the trials. The array was presented at random time intervals (about 36 presentations in $60 \mathrm{~s}$ ). The presentation duration varied in the range of 100-250 ms. This stimulus was used to engage attention on the right side of the fixation point.

\subsection{Procedure}

The subjects sat $114 \mathrm{~cm}$ away from the display, fixated on a central spot and, without moving their eyes, performed one of the 3 following tasks. In the attended condition, they had to discriminate and signal increments and decrements of the temporal frequency modulation of the grating displayed in the LVF. Some of the changes were easily detectable, others were more difficult, and often some were not detected at all. The subjects pressed one of two buttons with their right (increment) or left (decrement) thumbs. In the unattended condition, the subjects discriminated and signaled the presence and absence of the target letter among the distracters flashed on the RVF. The presentation duration varied during the course of the experiment to keep task accuracy between 70 and $80 \%$ of corrected responses. This procedure ensured that the search task was challenging throughout the experiment. Also in this case, the subjects pressed one of two buttons with their right (presence) or left (absence) thumbs. In the third condition (called passive), the subjects were asked to fixate on the central spot and to ignore both the grating and the letter array. Due to technical limitations, it was possible to record VEPs only from one hemifield; we selected the LVF to allow comparisons between the present study and previous investigations which used a similar setup (Di Russo and Spinelli, 1999a,b; Di Russo et al., 2001a).

A preliminary training period familiarized the subjects with the tasks while they kept a steady fixation. Eye movements were monitored through a camera. A zoom lens magnified one of the subject's eyes. Correct fixation was calibrated at the beginning of each recording sequence. The experimenter recorded the number of gaze shifts that occurred in each run and gave feedback to the subject during the experiment.

Steady-state VEPs were recorded during attended, unattended and passive conditions, alternated in successive blocks, with a short break in between. Each run lasted 60 $90 \mathrm{~s}$, during which about 20 changes of temporal frequency took place. VEPs to stimuli of various temporal frequencies were averaged in different files (up to 60 sums per run). 
Several blocks were run for each condition to collect VEPs to at least 360 stimulus repetitions.

\subsection{VEP recording and data analysis}

VEPs were recorded from two active scalp electrodes, one on the midline $(\mathrm{POz})$ and one on the right side (PO4) of the parietal-occipital areas, with $\mathrm{FCz}$ reference and $\mathrm{CPz}$ ground (10-10 system). Signals were amplified $(50,000$ fold), bandpass filtered $(1-30 \mathrm{~Hz})$ and digitized at 64 samples/period. The steady-state VEP waveform is roughly sinusoidal and is well described by the amplitude and phase of the second harmonic component (Campbell and Maffei, 1970). We used a method developed by Burr and Morrone in various studies (e.g. Morrone et al., 1987, 1993, 1996; Porciatti et al., 1992; Spinelli et al., 1994). The computer performed on-line Fourier analysis to calculate the amplitude and the phase of the second harmonic of the responses averaged (in the time domain) in packets of 20 sums. At least 18 packets (360 sums) were averaged (in the frequency domain) to obtain the final VEP. Standard deviations of amplitude and phase were calculated based on the scatter of the various packets contributing to the final VEP. The computer also averaged the electrical signals at a temporal frequency near that of the stimulus but not synchronous with it. This was taken as an index of noise to assess VEP reliability during the experiment. If the signal-to-noise ratio was very low, additional recording was performed. The signalto-noise ratio was calculated for each packet of 20 sums. Packets with low signal-to-noise ratios were eliminated offline (see Morrone et al., 1993 for details). The VEP latency was estimated from the slope of the regression line of VEP phases as a function of temporal frequency. The phase of the second harmonic was plotted in $\pi$ radians as a function of temporal frequency with the assumption that phase advances or retards regularly with temporal frequency. Thus, a multiple of $2 \pi$ radians is added to or subtracted from the raw data to produce the maximum orderliness (Regan, 1966; Spekreijse et al., 1977). The slope was calculated by least-squares fit after weighting each data point by its signal-to-noise ratio and by the inverse of the standard error of the mean of the phase variability.

Two separate repeated-factor ANOVAs were conducted on the amplitude and latency data. Task (attended, passive or unattended), channel (POz or $\mathrm{PO} 4)$ and temporal frequency (9 levels) comprised the factors for the amplitude analysis. For latency, task and channel were considered. An additional ANOVA was performed on the number of times the subjects moved their eyes away from the fixation point in the 3 tasks. $T$-tests were performed to evaluate the significance of the attentional costs and benefits. Post-hoc comparisons were based on Tukey's Honest Difference test and the alpha value was set at 0.01 .

\subsection{Control experiment}

The letter array on the RVF was flashed at random time intervals. Thus, it was desynchronized with respect to the grating contrast reversal and no contribution to the VEP was expected. However, to exclude its contribution, we recorded VEP to LVF grating as in the main experiment but with the contrast set at zero. In this condition, any possible contribution of the RVF flashed stimulus should be de-masked. Data were collected in 3 subjects.

\section{Results}

\subsection{Task accuracy}

In the attended condition, the subjects discriminated temporal frequency changes on average in $79 \%$ of the trials (range 69-91\%). In the unattended condition, letter discrimination among distracters was successful on average in $77 \%$ of the trials (range $67-87 \%$ ).

\subsection{Eye movements}

The median value of gaze shifts was 5 (range 2-15) in the attended condition, 7 (range 3-18) in the unattended condition, and 2 (range $0-8$ ) in the passive condition. The ANOVA showed that the effect of the condition was not significant $\left(F_{(2,24)}=3.4, P=0.054\right)$.

The median of the number of blinks was 9 (range 2-21) in the attended condition, 6 (range 1-17) in the unattended condition, and 11 (range 5-22) in the passive condition.

\subsection{VEP amplitude}

Fig. 1 reports the group's mean amplitudes recorded in the attended, passive and unattended conditions as a function of temporal frequency for the two electrode positions. Fig. 3a shows the mean amplitude (across temporal frequencies) as a function of the experimental conditions. When the subjects attended to the temporal frequency change, the VEP amplitudes were larger (mean 1.27 $\mu \mathrm{V}$ ) than those

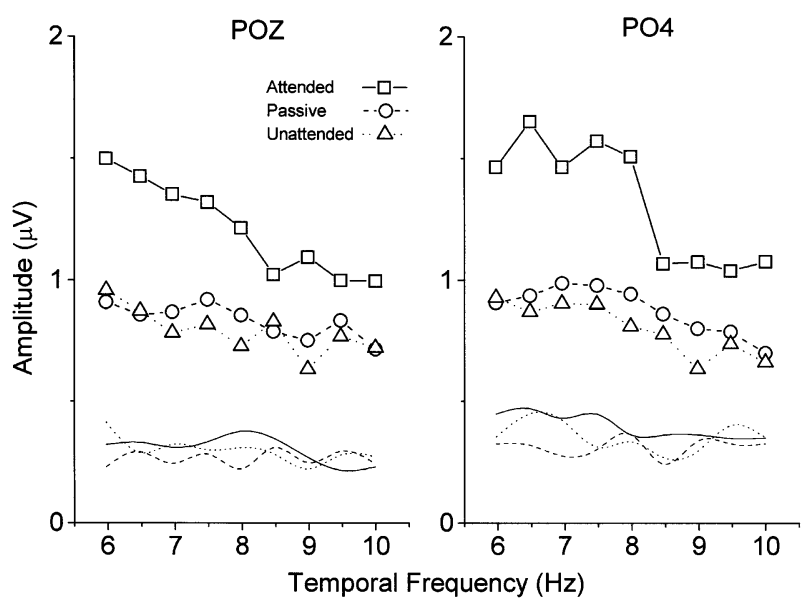

Fig. 1. Group VEP amplitude as a function of temporal frequency for midline (POz) and contralateral (PO4) electrode site in the 3 attention conditions. The lines with no symbols show the noise level. 
recorded from the same stimulus when the subjects attended to the stimulus in the opposite visual field $(0.81 \mu \mathrm{V})$ or passively observed the fixation point $(0.88 \mu \mathrm{V})$. The ANOVA showed that the main effects of task $\left(F_{(2,24)}=13.4, \quad P<0.001\right)$ and temporal frequency $\left(F_{(8,96)}=4.9, P<0.001\right)$ were significant. The main effect of the channel was not significant $(F<1)$. The interaction between task and temporal frequency was significant $\left(F_{(16,192)}=2.8, P<0.01\right)$. Post-hoc analyses showed that the attended amplitudes were larger than unattended and passive ones $(P<0.001)$ in the temporal frequency range 6-8 Hz.

\subsection{VEP latency}

Phase data, averaged across subjects separately for the 3 experimental conditions, are reported as a function of temporal frequency in Fig. 2. Estimated latencies of group data are reported in Fig. $3 \mathrm{~b}$ for the attended, passive and unattended conditions. These values were obtained calculating the individual latencies by regression and their values. The latency values obtained by regression of the group phase data (Fig. 2) were close to those obtained by averaging the individual latencies in all conditions $( \pm 2.7 \mathrm{~ms})$. The ANOVA on individual latencies showed a clear effect of attention $\left(F_{(2,24)}=83.7, P<0.001\right)$. Latencies in the attended condition $(122 \mathrm{~ms})$ were shorter $(P<0.001)$ than those recorded in passive $(141 \mathrm{~ms})$ and unattended conditions $(150 \mathrm{~ms})$. The latency in the passive condition was shorter than that recorded in the unattended condition $(P<0.005)$. The main effect of the channel and the interaction between factors were not significant.

\subsection{Costs and benefits}

Attentional benefits, i.e. differences between attended and neutral conditions, and attentional costs, i.e. differences

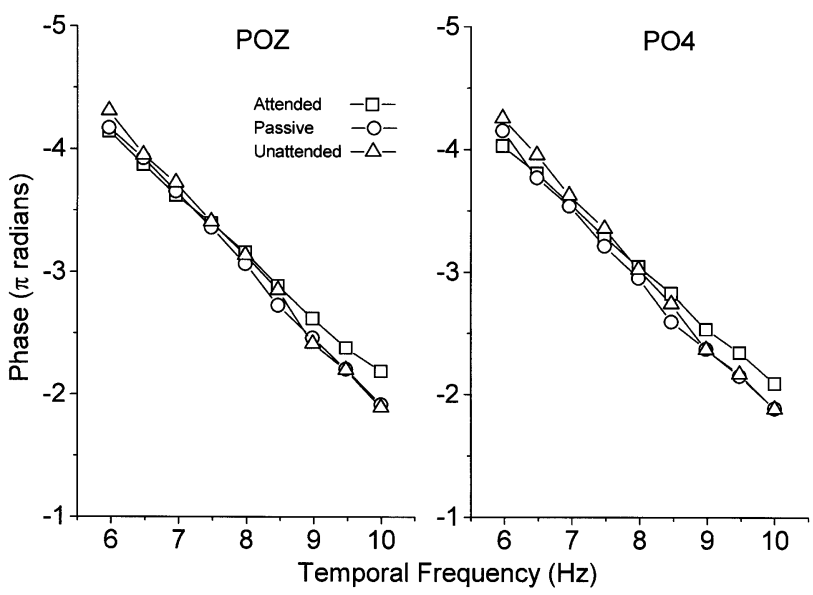

Fig. 2. Group VEP phases (in $\pi$ radians unit) as a function of temporal frequency for midline (POz) and contralateral (PO4) electrode sites in the 3 conditions. VEP latencies (reported in Fig. 3b) were derived from the slopes of the regression lines.
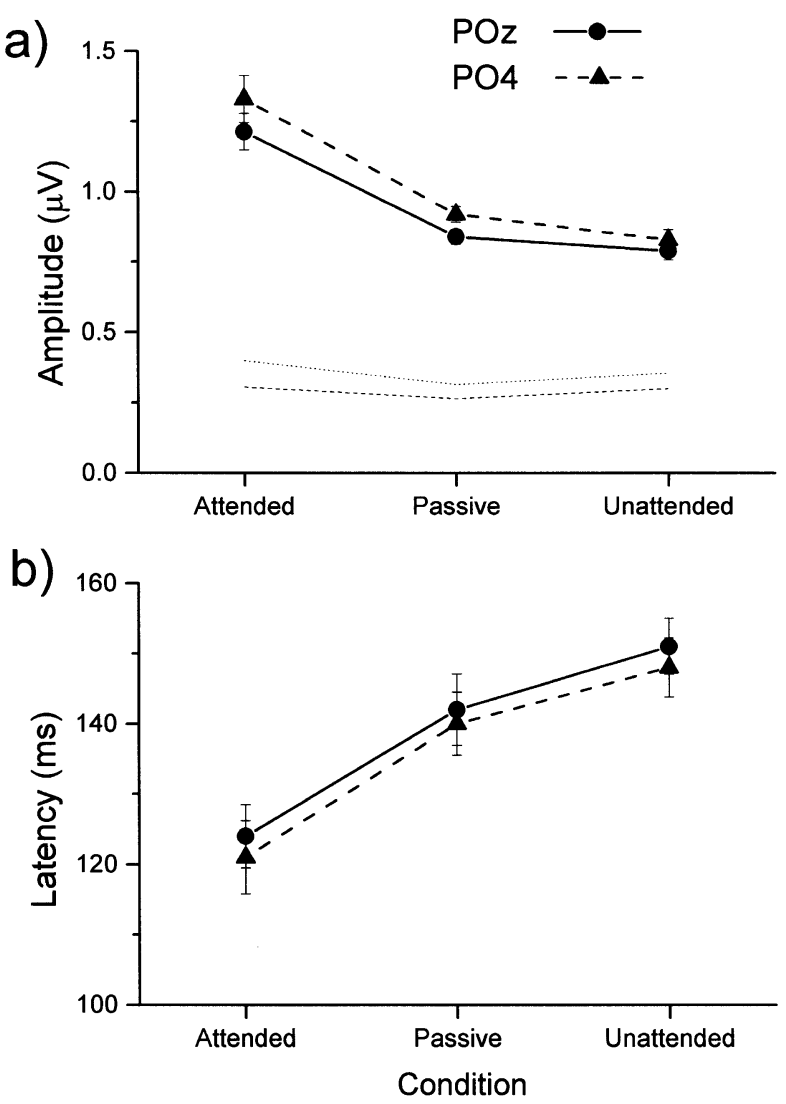

Fig. 3. Group VEP amplitude collapsed across temporal frequency (a) and group VEP latency (b) as a function of attention conditions for midline $(\mathrm{POz})$ and contralateral $(\mathrm{PO} 4)$ electrode sites. Vertical lines represent the standard error. The lines without symbols (a) show the noise level.

between neutral and unattended conditions, are reported in Fig. 4 for the amplitude and latency of steady-state VEPs. Benefits were significantly different from zero for both parameters $(P<0.0001)$. Costs were significant for latency $(P<0.005)$ and barely significant for amplitude $(P=0.077$ for $\mathrm{POz}$ and $P=0.046$ for PO4), suggesting a larger variance of the amplitude effect.

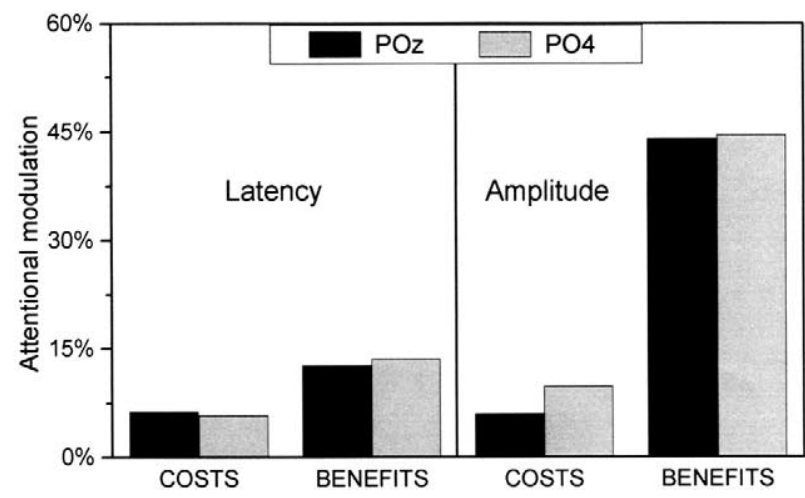

Fig. 4. Costs and benefits of the attention effect for latency and amplitude, recorded from the midline $(\mathrm{POz})$ and contralateral $(\mathrm{PO} 4)$ electrode sites. 


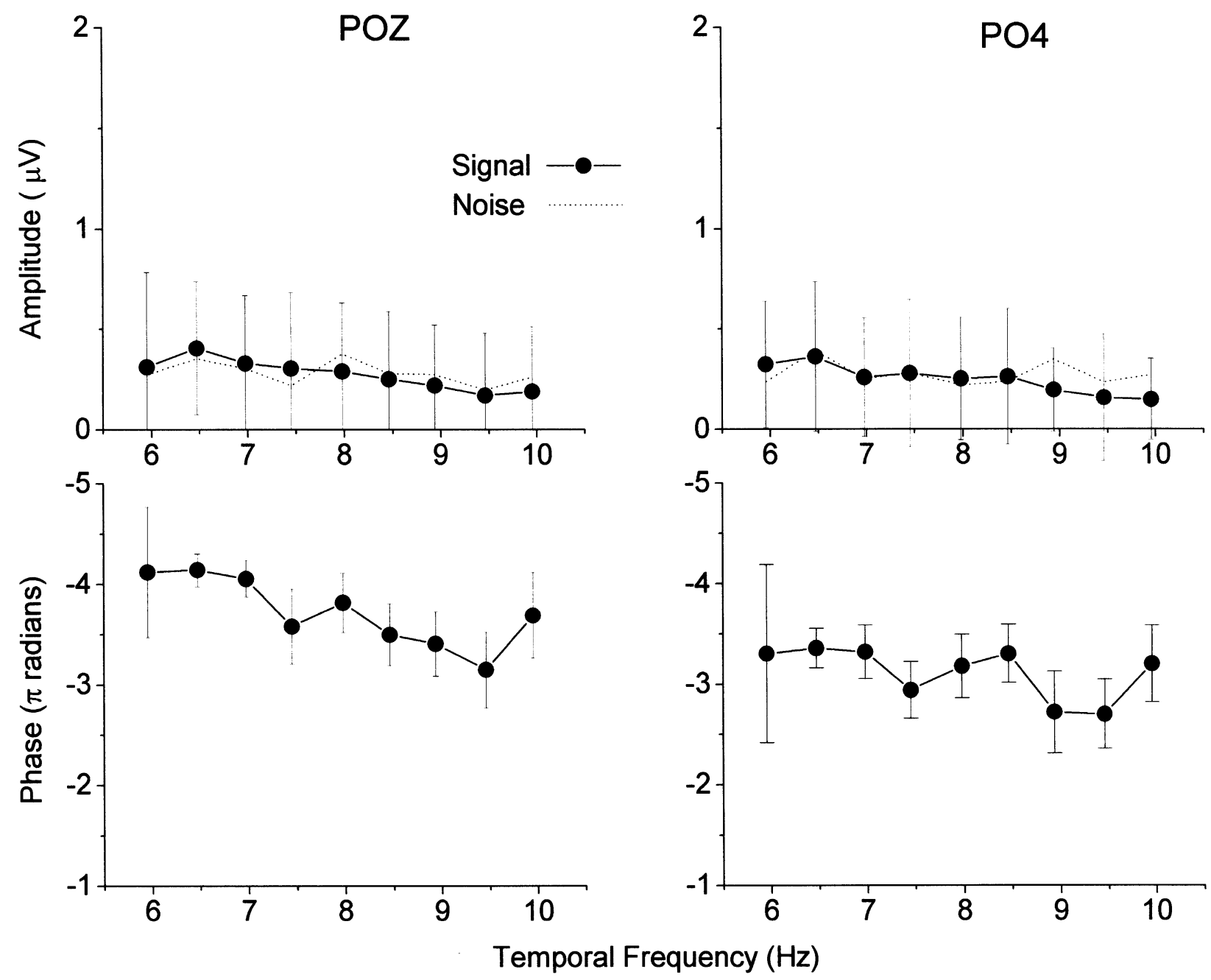

Fig. 5. Control condition. VEP amplitude with standard error and noise (top), and VEP phase (bottom) as a function of temporal frequency recorded from the midline (POz) and contralateral (PO4) electrode sites. Data represent the averages of 3 subjects.

\subsection{Control experiment}

Fig. 5 shows the mean amplitude and phase plotted as a function of temporal frequency with grating contrast set to zero. The VEP amplitude in this condition was at the level of noise for both electrodes and for all tested subjects. The phase was almost flat and had large variations. Overall, the electrophysiological response to the RVF flashed stimulus did not contribute to the response evoked by the grating reversal on the LVF.

\section{Discussion}

Steady-state VEPs are an excellent tool to study sustained attention. Brain activity is continuously averaged while the subject is attending to the stimulus across time. Activity is also averaged across space because different cortical areas contribute to the recorded waveform. Components, which are distinct in the transient VEP, are mixed up in steady- state VEPs and analyzed in the frequency rather than in the time domain.

When apparent latency is derived from VEPs (Spekreijse et al., 1977; Regan, 1989), it provides a measure of the averaged latency of the whole system in response to visual stimuli. It should be noted that the latency value in the attention condition in the present study (122 ms) is close to the peak latency typically measured for the transient P1 component (see Morrone et al., 1993). This suggests that brain areas V3, V3a and V4, responsible for P1 generation (Di Russo et al., 2001b), dominate the recorded activity; however, it does not exclude the contribution of other visual areas, such as V1 or V5.

In the present experiment, we measured the speeding up of visual processing when sustained, voluntary attention is directed to discriminate both evident and subtle changes of a stimulus permanently located in a portion of the visual field. We compared the responses evoked by the same physical stimulus in 3 different conditions. In one condition, the subject's attention was directed towards the stimulus and, 
for about $1 \mathrm{~min}$, he/she discriminated and signaled temporal frequency increments and decrements. In a second condition, the subject's attention was directed towards another stimulus, located in the opposite space. Again, for about 1 min the subject searched for and signaled the presence or absence of a target letter among distracters. These two experimental conditions were identical in terms of stimuli displayed in the visual field and motor response required of the subject; also task difficulty was similar. Experimental conditions differed only in the allocation of attention, which was toward the recording stimulus in one case, and away from it in the second case. In the third experimental condition, the visual stimuli displayed were identical, but the subject was not involved in a demanding task. Thus, attention was moved away from the recording stimulus, but in a less active way. This neutral condition offers a measurement of brain activity evoked by the stimulus while the subject remains fixated on the fixation point for $1 \mathrm{~min}$, ignoring lateral stimuli (however, uncontrolled shifts of attention may occur towards one or the other stimulus). The VEP amplitude and phase in the neutral condition represented a baseline against which we measured the effect of allocating (directing) attention to the stimulus or moving attention away from it.

An enhanced VEP amplitude with respect to the baseline reflects the benefits of voluntary attention allocation. The smaller amplitudes in the unattended with respect to the baseline condition reflect costs that are hardly significant. Similar results were obtained by Mangun and Buck (1999). They reported attentional benefits and no costs for the P1 component in a transient VEP study using the sustained attention paradigm. Contrasting findings (Luck et al., 1994), showing only attentional costs on P1, may be due to the use of a different (trial-by-trial cueing) paradigm (Mangun and Buck, 1999).

Since it shows significant costs and benefits, latency seems to be very sensitive for measuring attentional involvement. This is a new result. Except for our previous studies, where we did not test a neutral condition, there are no reports of attentional modulation of the latency of early components in the literature. Regarding late components latency, Mangun and Buck (1999) found a substantial cost (but no benefit) for the late positive deflection (mean peak around $370 \mathrm{~ms}$ ).

The present results reinforce the notion that latency may be the key of a priority-based attention mechanism acting at an early level (Di Russo and Spinelli, 1999a). The faster visual response wins the competition for processing with respect to other brain signals. Processing priority, marked by a short VEP latency in the present experiment, was associated with an intense stimulus consciousness; it is well known that, in this condition, discrimination improves (e.g. Lee et al., 1999), and visual processing (Carrasco and McElree, 2001) and motor reactions (e.g. Posner et al., 1980) are faster. The slower stimulus processing measured here in the passive condition was associated with reduced consciousness of the stimulus itself, as reported by the subjects at the end of the experiment, during an informal interview. When attention was actively shifted away from the stimulus, the slowest latency was associated with a very low (null, for most of the time) consciousness of the recording stimulus, and we know that this condition produces poor discrimination ability and slower reaction times.

At present, we can only speculate about the mechanisms involved in the attentional effect described here. Voluntary allocation of attention may be mediated by feedback projections from higher-order areas in the frontal and parietal cortex to the visual cortex (Kastner and Ungerleider, 2000; Martínez et al., 2001a; Di Russo et al., 2002). Topdown bias may modulate automatic gain control mechanisms at an early stage (Di Russo et al., 2001a). This produces speeding up and enhancing of early processing of the attended stimulus.

\section{Acknowledgements}

This study was supported by a MURST grant to D. Spinelli.

\section{References}

Bernardete EA, Kaplan E, Knight BW. Contrast gain control in the primate retina: $\mathrm{P}$ cells are not $\mathrm{X}$-like, some $\mathrm{M}$ cells are. Vis Neurosci 1992;8:483-486.

Campbell FW, Maffei L. Electrophysiological evidence for the existence of orientation and size detectors in the human visual system. J Physiol (Lond) 1970;192:345-358.

Carandini M, Heeger DJ. Summation and division by neurons in primate visual cortex. Science 1994;264:1333-1335.

Carrasco M, McElree B. Covert attention accelerates the rate of visual information processing. Proc Natl Acad Sci USA 2001;98:5363-5367.

Di Russo F, Spinelli D. Electrophysiological evidence for an early attentional mechanism in visual processing in humans. Vis Res 1999a;39:2975-2985.

Di Russo F, Spinelli D. Spatial attention has different effects on the magnoand parvo-cellular pathways. NeuroReport 1999b;10:2755-2762.

Di Russo F, Spinelli D, Morrone MC. Automatic gain control contrast mechanisms are modulated by attention in humans: evidence from visual evoked potentials. Vis Res 2001a;4:2435-2447.

Di Russo F, Martínez A, Sereno MI, Pitzalis S, Hillyard SA. The cortical sources of the early components of the visual evoked potential. Hum Brain Mapping 2001b;15:95-111.

Di Russo F, Martínez A, Hillyard SA. Source analysis of cortical generators involved in visuo-spatial attention. Cereb Cortex 2002 in press.

Eimer M. ERP modulations indicate the selective processing of visual stimuli as a result of transient and sustained spatial attention. Psychophysiology 1996;33:13-21.

Hillyard SA, Annlo-Vento L. Event-related brain potentials in the study of visual selective attention. Proc Natl Acad Sci USA 1998;95:781-787.

Hillyard SA, Mangun GR. Sensory gating as a physiological mechanism for visual selective attention. Electroenceph clin Neurophysiol Suppl 1987;40:61-67.

Hillyard SA, Mangun GR, Woldorff MG, Luck SJ. Neural systems mediating selective attention. In: Gazzaniga MS, editor. The cognitive neurosciences, Cambridge, MA: MIT Press, 1995. pp. 665-681. 
Hillyard SA, Vogel EK, Luck SJ. Sensory gain control (amplification) as a mechanism of selective attention: electrophysiological and neuroimaging evidence. Phil Trans R Soc Lond B Biol Sci 1998;353:1257-1267.

Kastner S, Ungerleider LG. Mechanisms of visual attention in the human cortex. Annu Rev Neurosci 2000;23:315-341.

Lee DK, Itti L, Koch C, Braun J. Attention activates winner-take-all competition among visual filters. Nat Neurosci 1999;2:375-381.

Luck SJ, Hillyard SA, Mouloua M, Woldorff MG, Clark VP, Hawkins HL. Effect of spatial cuing on luminance detectability: psychophysical and electrophysiological evidence for early selection. J Exp Psychol Hum Percept Perform 1994;20:1000-1014.

Mangun GR, Buck LA. Sustained visual-spatial attention produces cost and benefits in response time and evoked neural activity. Neuropsychologia 1999;36:189-200.

Martínez A, Di Russo F, Annlo-Vento L, Sereno MI, Buxton R, Hillyard SA. Putting spatial attention on the map: timing and localization of stimulus selection processes in striate and extrastriate visual areas. Vis Res 2001a;41:1437-1457.

Martínez A, Di Russo F, Anllo-Vento L, Hillyard SA. Electrophysiological analysis of cortical mechanisms of selective attention to high and low spatial frequencies. Clin Neurophysiol 2001b;112:1980-1998.

Morgan ST, Hansen JC, Hillyard SA. Selective attention to stimulus location modulates the steady-state visual potential. Proc Natl Acad Sci USA 1996;93:4770-4774.

Morrone MC, Burr DC, Speed H. Cross-orientation inhibition in cat is GABA mediated. Exp Brain Res 1987;67:635-644.

Morrone MC, Burr DC, Fiorentini A. Development of infant contrast sensitivity to chromatic stimuli. Vis Res 1993;33:2535-2552.

Morrone MC, Fiorentini A, Burr DC. Development of the temporal properties of visual evoked potentials to luminance and colour contrast in infant. Vis Res 1996;36:3141-3155.

Müller M, Hillyard SA. Concurrent recording of steady-state and transient event-related potentials as indices of visual-spatial selective attention. Clin Neurophysiol 2000;111:1544-1552.

Müller MM, Picton TW, Valdes-Sosa P, Riera P, Teder-Sälejärvi AW, Hillyard SA. Effects of spatial selective attention on the steady-state visual evoked potential in the $20-28 \mathrm{~Hz}$ range. Cog Brain Res 1998a;6:249-261.

Müller MM, Teder-Sälejärvi AW, Hillyard SA. The time course of cortical facilitation during cued shifts of spatial attention. Nat Neurosci 1998b;1:631-634.

Porciatti V, Burr DC, Morrone MC, Fiorentini A. The effects of ageing on the pattern electroretinogram and visual evoked potential in humans. Vis Res 1992;32:1199-1209.

Posner MI. Attention in cognitive neuroscience: an overview. In: Gazzaniga MS, editor. The cognitive neurosciences, Cambridge, MA: MIT Press, 1995. pp. 615-624.

Posner MI, Snyder CR, Davidson BJ. Attention and the detection of signals. J Exp Psychol 1980;109:160-174.

Proverbio AM, Mangun GR. Electrophysiological and behavioral "costs" and "benefits" during sustained visual-spatial attention. Int J Neurosci 1994;79:221-233.

Regan D. Some characteristics of average steady-state and transient responses evoked by modulated light. Electroenceph clin Neurophysiol 1966;20:238-248.

Regan D. Human brain electrophysiology: evoked potentials and evoked magnetic fields in science and medicine, New York: Elsevier, 1989.

Shapley RM, Victor JD. The effect of contrast on the transfer properties of cat retinal ganglion cells. J Physiol (Lond) 1978;285:275-298.

Shapley RM, Victor J. How the contrast gain control modifies the frequency responses of cat retinal ganglion cells. J Physiol (Lond) 1981;318:161179.

Spekreijse H, Estevez O, Reits D. Visual evoked potentials and the physiological analysis of visual processes in man. In: Desmedt JE, editor. Visual evoked potentials in man: new development, Oxford: Clarendon Press, 1977. pp. 16-89.

Spinelli D, Burr DC, Morrone MC. Spatial neglect is associated with increased latencies of visual evoked potentials. Vis Neurosci 1994;11:909-918.

Vogel EK, Luck SJ. The visual N1 component as an index of a discrimination process. Psychophysiology 2000;37:190-203. 